\title{
Organization of the Presynaptic Active Zone by ERC2/ CAST1-Dependent Clustering of the Tandem PDZ Protein Syntenin-1
}

\author{
Jaewon Ko, ${ }^{1}$ Chan Yoon, ${ }^{1}$ Giovanni Piccoli, ${ }^{2}$ Hye Sun Chung, ${ }^{3}$ Karam Kim, ${ }^{1}$ Jae-Ran Lee, ${ }^{1}$ Hyun Woo Lee, ${ }^{1}$ Hyun Kim, ${ }^{3}$ \\ Carlo Sala, ${ }^{2}$ and Eunjoon $\mathrm{Kim}^{1}$ \\ ${ }^{1}$ National Creative Research Initiative Center for Synaptogenesis and Department of Biological Sciences, Korea Advanced Institute of Science and \\ Technology, Daejeon 305-701, Korea, ${ }^{2}$ Consiglio Nazionale delle Ricerche, Institute of Neuroscience, Cellular and Molecular Pharmacology, Department of \\ Pharmacology, University of Milan, 20129 Milan, Italy, and ${ }^{3}$ Department of Anatomy and Division of Brain Korea 21 Biomedical Science, College of \\ Medicine, Korea University, Seoul 136-705, Korea
}

Presynaptic active zones contain a cytoskeletal matrix called the CAZ, which is thought to play a critical role in the regulation of active zone formation and neurotransmitter release. Recent studies have identified several CAZ components, but little is known about how they contribute to the molecular organization of active zones. Here, we report a novel PDZ [postsynaptic density-95/Discs large/zona occludens-1] interaction between the CAZ protein ERC2/CAST1 and the tandem PDZ protein syntenin-1, which is known to associate with diverse synaptic proteins, including glutamate receptor subunits, SynCAM, and $\beta$-neurexin. This interaction promotes the localization of syntenin-1 at presynaptic ERC2 clusters. In addition to the PDZ interaction, multimerization of both ERC2 and syntenin-1 mediates syntenin-1 clustering. These results suggest that ERC2 promotes presynaptic syntenin-1 clustering by two distinct mechanisms and that syntenin-1 may contribute to the molecular organization of active zones by linking ERC2 and other CAZ components to diverse syntenin-1-associated synaptic proteins.

Key words: ERC; CAST; syntenin; PDZ; multimerization; presynaptic regulation

\section{Introduction}

Active zones are specialized regions of the presynaptic plasma membrane where neurotransmitter vesicles dock, fuse, and release neurotransmitters. Active zones are characterized by the presence of an electron-dense matrix of proteins termed the presynaptic cytomatrix at the active zone (CAZ). The CAZ contains various multidomain proteins that are thought to organize active zones, recruit synaptic vesicles, and regulate neurotransmitter release (Dresbach et al., 2001; Rosenmund et al., 2003; Sudhof, 2004; Zhen and Jin, 2004; Ziv and Garner, 2004). Known CAZ proteins include Piccolo/aczonin, Bassoon, RIM, Munc13, ERC/ CAST, and liprin- $\alpha$. But it is primarily unknown how they contribute to the structural and functional organization of presynaptic active zones.

The ERC family of CAZ proteins contains two known members: ERC1/ELKS/Rab6IP2/CAST2 and ERC2/CAST1 (Nakata et al., 1999; Monier et al., 2002; Ohtsuka et al., 2002; Wang et al.,

\footnotetext{
Received June 9, 2005; revised Nov. 22, 2005; accepted Nov. 23, 2005.

This work was supported by the National Creative Research Initiative Program of the Korean Ministry of Science and Technology (E.K.), the Giovanni Armenise-Harvard Foundation Career Development Program and European Community (LSHM-CT-2004-511995, SYNSCAFF to C.S.), and the 21st Century Frontier Research and Development Program in Neuroscience of the Korean Ministry of Science and Technology (M103KV010018-03K2201-01820 to H.K.).

Correspondence should be addressed to Dr. Eunjoon Kim, Department of Biological Sciences, Korea Advanced Institute of Science and Technology, Daejeon 305-701, Korea. E-mail: kime@kaist.ac.kr. DOI:10.1523/JNEUROSCI.4475-05.2006

Copyright $\odot 2006$ Society for Neuroscience $\quad 0270-6474 / 06 / 260963-08 \$ 15.00 / 0$
}

2002; Deguchi-Tawarada et al., 2004). ERC1 has two splice variants, ubiquitous ERC1a and brain-specific ERC1b, whereas no splice variants are known for the brain-specific ERC2. ERC1b and ERC2 share the same C-terminal PDZ [postsynaptic density-95 (PSD-95)/Discs large/zona occludens-1] domain-binding motif. ERC interacts with other CAZ proteins including RIM, Piccolo, Bassoon, and liprin- $\alpha$ (Ohtsuka et al., 2002; Wang et al., 2002; Ko et al., 2003b; Takao-Rikitsu et al., 2004). Functionally, the interaction of ERC2 with RIM1 and Bassoon/Piccolo has been shown to regulate neurotransmitter release (Takao-Rikitsu et al., 2004). These previous reports suggest that ERC regulates CAZ assembly and presynaptic function, but molecular mechanisms underlying these functions are not well understood.

Syntenin is a family of tandem PDZ proteins with two known members: syntenin-1 and syntenin-2. Syntenin was originally isolated as binding partners of syndecan (Grootjans et al., 1997), a cell surface heparan sulfate proteoglycan. The PDZ domains of syntenin interact with a variety of membrane and cytosolic proteins including glutamate receptor subunits (AMPA, kainate, and metabotropic), $\beta$-neurexin, SynCAM, and Rab5 (Biederer et al., 2002; Hirbec et al., 2002, 2003; Enz and Croci, 2003; Tomoda et al., 2004). Accordingly, syntenin has been implicated in various cellular processes including cell adhesion, synaptic transmission, membrane-cytoskeleton organization, signaling, trafficking, and gene expression.

Here, we report ERC2 associates with and induces presynaptic 
clustering of syntenin-1 by two distinct mechanisms: PDZ interaction and protein multimerization. These mechanisms may contribute to the organization of active zones.

\section{Materials and Methods}

Yeast two hybrid. ERC2 (amino acids 833-954 and 833-957), ERC1b (881-992), syndecan-2 (170-201), ephrin-B2 (258-336), and EphA7 (915-998) were subcloned into pBHA (bait vector). pGAD10 (prey vector; Clontech, Mountain View, CA) constructs were as follows: syntenin-1 deletions (see Fig. $1 A$ ); syntenin-2 $\alpha$ (amino acids 1-292); RIM1 $\alpha$ (597-707); and RIM2 $\beta$ (452-582).

cDNA constructs. Rat syntenin-1 was subcloned into pFLAG-CMV2 (Sigma, St. Louis, MO) and pEGFP-C1 (Clontech). Human syntenin- $2 \alpha$ and human ERC2 were subcloned into pFLAG-CMV2 and pCDNA3-HA (Invitrogen, San Diego, CA), respectively. For pull down, full-length syntenin-1 and ECR2 (amino acids 948-957) were subcloned into pET32a and pGEX4T-1. EGFP-ERC2 and EGFPERC1b constructs have been described previously (Ko et al., 2003b).

In situ hybridization. In situ hybridization was performed as described previously (D. Kim et al., 2003). Hybridization probes were prepared from pGEM7zf containing rat ERC1b (nucleotides 2761-3356; GenBank accession number AF541926), ERC2 (305-699; GenBank accession number AF541925), and syntenin-1 (1081-1680; GenBank accession number AF248548). ${ }^{35}$ S-labeled antisense riboprobes were prepared using Riboprobe System (Promega, Madison, WI).

Antibodies. Immunogens were glutathione S-transferase (GST)syntenin-1 (amino acids 1-300; 1308 rabbit; 1313 guinea pig) and GSTERC1b (429-700; 1395 rabbit; 1398 guinea pig). The following antibodies have been described previously: ERC2 1292 (Ko et al., 2003b), EGFP 1173 (Ko et al., 2003a), Piccolo 1203 (S. Kim et al., 2003), KIF1A 1131 (Shin et al., 2003), and S-SCAM 1146 (Mok et al., 2002). The following antibodies were purchased: $\alpha$-tubulin, synaptophysin, and FLAG (Sigma); Myc 9E10 and rabbit hemagglutinin (HA) (Santa Cruz Biotechnology, Santa Cruz, CA); and RIM1 (Transduction Laboratories, Lexington, KY).

Neuron culture, transfection, and immunocytochemistry. Primary hippocampal cultures were prepared from embryonic day 18-19 rat hippocampi. Neurons were transfected using a mammalian transfection kit (Invitrogen), fixed with cold 100\% methanol, and stained as described previously (Ko et al., 2003a).

Image acquisition and quantitative analysis. Confocal images were analyzed using the MetaMorph software (Universal Imaging Corporation, West Chester, PA). Images of distal thin neurites (probably axons) of cultured neurons from three to five independent experiments were captured for analysis. A cluster was defined as a discrete region of immunoreactivity with an average fluorescence intensity at least 10 -fold higher than that in background regions. Colocalization between two puncta was defined as an overlap of $>50 \%$ of each region, and colocalization analyses were performed blind. Approximately 30-50 clusters were analyzed per frame, and the means from multiple individual frames were averaged to obtain a population mean and SEM.

\section{Results}

In vitro interaction between $\mathrm{ERC}$ and syntenin

ERC1b and ERC2 share a class II PDZ-binding C-terminal motif (Kim and Sheng, 2004) that interacts with RIM (Ohtsuka et al.,
2002; Wang et al., 2002). Because RIM may not be the only (or major) binding partner of ERC, we searched for additional PDZ proteins using the ERC2 C terminus (amino acids 833-957) as bait in yeast two-hybrid screens. Of 80 positive clones, 42 encoded overlapping fragments of syntenin-1. Notably, RIM was not isolated from the screen, and syntenin-1 was the only PDZ protein among the positive clones. However, ERC2 interacted equally with syntenin-1 and RIM1 in yeast two-hybrid assays (see below). Thus, the lack of RIM1 in prey clones may simply reflect the scarcity of RIM1 cDNA in our yeast two-hybrid library.

In yeast two-hybrid assays, ERC2 lacking the last three residues lost interaction with syntenin-1, and in syntenin-1, both PDZ domains were required for ERC2 binding (Fig. $1 \mathrm{~A}$ ). A point mutation in PDZ2 (G212D) but not in PDZ1 (G128E) eliminated ERC2 binding (Fig. $1 \mathrm{~B}$ ), suggesting that PDZ2 mediates ERC2 binding. ERC2 did not interact with PDZ domains from Piccolo, GRIP1, PSD-95, or LIN-7 (data not shown). Syntenin-2 also weakly interacted with ERC2 (Fig. $1 B$ ), similar to $\beta$-neurexin, which interacts with both syntenins (Koroll et al., 2001). Point mutations in the last four residues of ERC2 differentially affected its binding to syntenins and RIMs (Fig. 1C), suggesting that these interactions use slightly different mechanisms.

GST-syntenin-1 pulled down only the ERC2 deletions containing the $\mathrm{C}$ terminus (amino acids 1-957 and 773-957) (Fig. 2A). In addition, GST ERC2 (amino acids 948-957), but not GST alone, pulled down hexahistidine-tagged, full-length syntenin-1 fusion proteins, indicating that the two proteins interact directly (Fig. 2 B). In heterologous cells, ERC2 and ERC1b formed a complex with syntenin-1 and syntenin-2 (Fig. $2 C--F$ ), but not with 


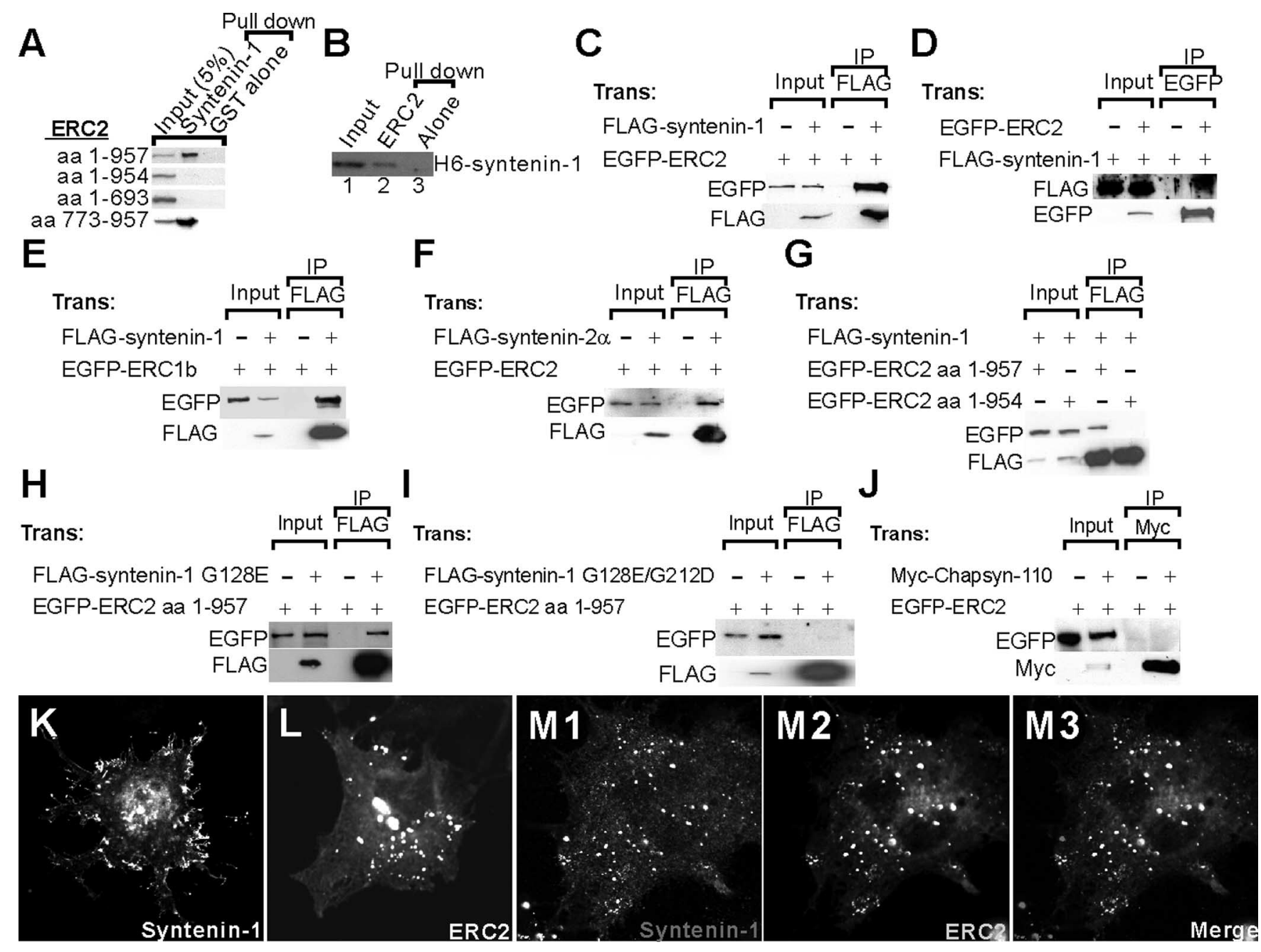

Figure 2. Interaction of ERC with syntenin in pull-down, coimmunoprecipitation, and coclustering assays. A, Pull down of ERC2 by syntenin-1. EGFP-ERC2 variants expressed in HEK293T cells were pulled down by GST-syntenin-1 and immunoblotted. $\boldsymbol{B}$, Pull down of syntenin-1 by ERC2. Hexahistidine (H6)-tagged, full-length syntenin-1 fusion proteins were pulled down by GST-ERC2 (amino acids 948 -957) but not by GST alone. Input, 10\%. C-F, Coimmunoprecipitation between ERC- and syntenin-family proteins in HEK293T cells. Lysates of HEK293T cells singly or doubly transfected with the indicated ERC and syntenin constructs were immunoprecipitated and immunoblotted with the antibodies indicated. $\boldsymbol{C}$ and $\boldsymbol{D}$ are the same immunoprecipitation reactions in opposite directions. Input, 5\%. G-I, The coimmunoprecipitation between ERC2 and syntenin-1 requires the C terminus of ERC2 and the PDZ2 domain of syntenin-1. Lysates of HEK293T cells singly or doubly transfected with the indicated ERC2 and syntenin-1 variants (ERC2 amino acids 1-954 lacking the last 3 PDZ-binding residues; G128E, a point mutation in PDZ1; G128E/G212D, point mutations in both PDZ1 and PDZ2) were precipitated and immunoblotted with the antibodies indicated. Input, 5\%.J, Lack of coimmunoprecipitation between ERC2 and Chapsyn-110/PSD-93 (a negative control PDZ protein). $\boldsymbol{K}-\boldsymbol{M}$, Redistribution of syntenin-1 to ERC2 clusters. COS-7 cells expressing FLAG-syntenin-1 (K), EGFP-ERC2 (L), or both (M1-M3) were visualized with FLAG (syntenin-1) and EGFP (ERC2) antibodies. Trans, Transfected; IP, immunoprecipitated.

chapsyn-110/PSD-93 (control) (Fig. 2J). C-terminal deletion in the ERC2 and PDZ2 mutation in syntenin-1 eliminated the interaction (Fig. 2G,I). In a coclustering assay, syntenin-1 was redistributed from focal adhesion-like sites to ERC2 clusters (Fig. $2 K-M$ ). Together, these results indicate that ERC- and syntenin-family proteins associate through the canonical PDZ interaction.

\section{Overlapping distribution patterns and biochemical} association between ERC and syntenin in the brain We first compared the mRNA distribution patterns of ERC1b, ERC2, and syntenin-1 (Fig. 3A-C). ERC1b and ERC2 mRNAs were widely expressed in rat brain regions at postnatal days 3,7 , and 12 (Fig. $3 A, B$ ). However, at the adult stage (6 weeks), ERC1b and ERC2 showed an interesting segregation: ERC1b mainly distributed to the hippocampus and cerebellum, whereas ERC2 was localized in forebrain regions including the olfactory bulb, cortex, hippocampus, and medial habenular nucleus of the thalamus
(Fig. 3A,B). However, syntenin-1 distributed to various brain regions without any obvious segregation throughout postnatal stages (Fig. $3 C$ ), consistent with a recent report (Ohno et al., 2004). Thus, syntenin-1 may associate with ERCs in specific adult brain regions (i.e., syntenin-1 mainly associates with ERC1b in the cerebellum; with ERC2 in the olfactory bulb, cortex, and medial habenular; and with both ERC1b and ERC2 in the hippocampus).

Next, we generated ERC and syntenin- 1 antibodies (1308 and 1313, specific for syntenin-1; 1395 specific for both ERC1b and ERC2; 1398 specific for ERC1b) (Fig. 4A-C). Immunoblot analysis revealed that both syntenin-1 and ERC are widely expressed in regions including the cortex, cerebellum, hippocampus, and striatum (Fig. 4D). The expression levels of syntenin-1, ERC1b, and ERC2 increased during postnatal development (Fig. 4E). Syntenin-1 distributed to various subcellular regions including the crude synaptosomal (P2), cytosolic (S3), and light membrane (P3) fractions, and ERC1b and ERC2 were mainly detected in the 


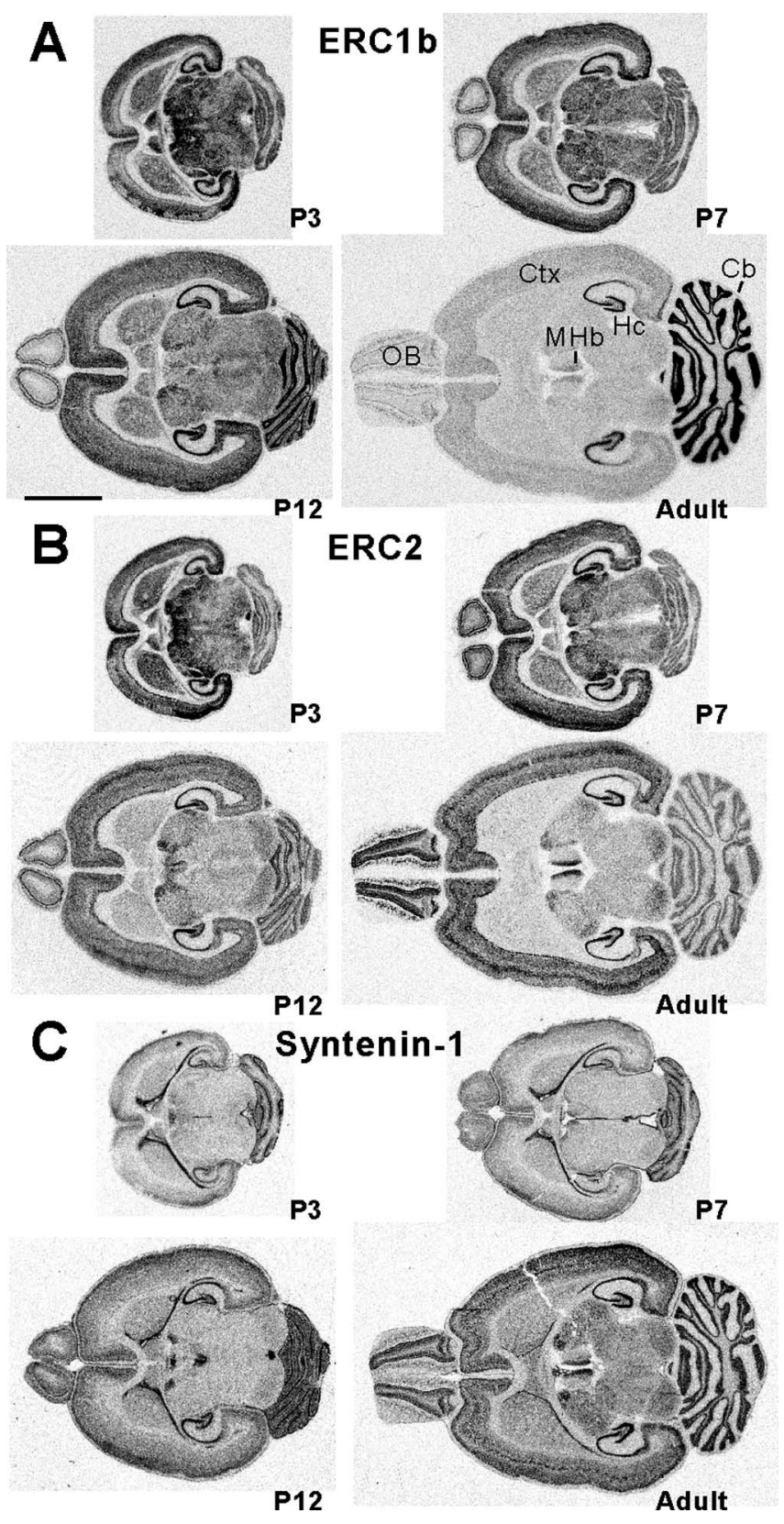

Figure 3. Overlapping mRNA expression patterns of ERC $1 b, E R C 2$, and syntenin- 1 in the brain. $\boldsymbol{A}-\boldsymbol{C}$, mRNA distribution patterns. Sections from postnatal day 3 (P3), P7, P12, and adult (6 weeks) rat brains were probed with ERC1b, ERC2, and syntenin-1 CRNAs. Scale bar, $6 \mathrm{~mm} .0 \mathrm{~B}$, Olfactory bulb; Ctx, cerebral cortex; Hc, hippocampus; MHb, medial habenular nucleus of the thalamus; $c b$, cerebellum.

P2 fraction, although ERC1b was more cytosolic than ERC2 because it was present in S3 and LS2 (synaptosomal cytosol) fractions (Fig. $4 F$ ). These results suggest that ERCs and syntenin-1 have overlapping regional, developmental, and subcellular expression patterns. In pull-down assays with rat brain lysates, GST-syntenin-1 brought down ERC2 and ERC1b but not negative controls (RIM1, PSD-95, and S-SCAM) (Fig. 4G,H). In coimmunoprecipitation experiments with the same lysates, syntenin-1 coprecipitated with ERC2 and ERC1b (Fig. 4I,J). Notably, however, syntenin-1 did not coimmunoprecipitate with RIM1 (Fig. 4I). These results suggest that ERC associates with syntenin-1 in the brain.
ERC2 promotes presynaptic clustering of syntenin-1

Our coclustering and biochemical fractionation data (Figs. $2 \mathrm{~K}-\mathrm{M}, 4 F$ ) and previous reports (Ohtsuka et al., 2002; Wang et al., 2002) indicate that ERC2 is active zone specific, whereas syntenin-1 is partially synaptic, suggesting that ERC2 may regulate presynaptic syntenin-1 clustering. When expressed alone in cultured neurons, syntenin-1 partially colocalized with Piccolo and ERC2; $18.3 \pm 3.4 \%(n=18)$ of Piccolo and $22.2 \pm 2.8 \%(n=$ 23 ) of ERC2 clusters were syntenin-1 positive (Fig. $5 A, B, F$ ). Importantly, when syntenin-1 was coexpressed with ERC2, which is targeted to presynaptic sites by itself (Ohtsuka et al., 2002; Ko et al., 2003b), syntenin-1 was mostly redistributed to ERC2 clusters $(69.6 \pm 7.2 \%$ of ERC2 clusters were syntenin- 1 positive; $n=13$; ${ }^{\star} p<0.0001$ compared with syntenin- 1 alone) (Fig. $5 C, F$ ). These results suggest that ERC2 promotes presynaptic syntenin-1 clustering. Notably, syntenin-1 was distributed in both axons and dendrites (supplemental Fig. 1, available at www.jneurosci.org as supplemental material), suggesting that syntenin-1 has additional functions in dendrites. Consistent with these findings, a recent report showed that syntenin-1 is involved in the regulation of dendritic protrusions (Hirbec et al., 2005).

ERC2-dependent presynaptic syntenin-1 clustering involves both the PDZ interaction and ERC2 multimerization

Surprisingly, ERC2 amino acids 1-954, which lack ERC2 binding, induced a high-level syntenin clustering $(73.8 \pm 5.7 \%$; $n=17$; ${ }^{*} p<0.0001$ compared with syntenin-1 alone) (Fig. 5D,F) similar to that induced by full-length ERC2. In addition, we did not observe a reduction in either the area $(2.51 \pm 0.29$ for ERC2 amino acids $1-954, n=17, p=0.0579 ; 3.33 \pm 0.30$ for ERC2 amino acids $1-957, n=13$ ) or the immunofluorescence intensity (180.55 \pm 6.70 for ERC2 amino acids $1-954, n=17, p=0.5682$; $185.18 \pm 4.39$ for ERC2 amino acids $1-957, n=13$ ) of the syntenin-1 clusters. A possibility is that ERC2 amino acids 1-954, which can be targeted to presynaptic sites by themselves (Ohtsuka et al., 2002; Ko et al., 2003b), might have recruited endogenous ERC2 and/or ERC1b by multimerization (DeguchiTawarada et al., 2004), promoting syntenin-1 clustering. This could be tested by examining whether an ERC2 mutant lacking both PDZ interaction and multimerization is capable of promoting syntenin-1 clustering.

To obtain such ERC2 mutant, we tested coimmunoprecipitation between two differentially tagged ERC2 deletion variants (Fig. 5G-I). In HEK293T cells, EGFP-ERC2 amino acids 1-693, but not 1-957 or 1-954, lost their interaction with HA-ERC2 full length. When expressed in neurons, ERC2 amino acids 1-693, which are targeted to presynaptic sites by themselves (Ko et al., 2003 b), did not promote syntenin-1 clustering $(28.7 \pm 3.2 \%$; $n=$ $12 ; p=0.1599$ compared with syntenin-1 alone) (Fig. $5 E, F)$. These results suggest that ERC2 promotes presynaptic syntenin-1 clustering through two distinct mechanisms: PDZ interaction and ERC multimerization.

\section{ERC2-dependent syntenin-1 clustering involves syntenin-1 multimerization}

Next, we tested syntenin-1 PDZ domain mutants. The syntenin-1 PDZ1 mutant (G128E) showed a high-level ERC2-dependent clustering ( $75.9 \pm 4.3 \%$ of ERC2 clusters were syntenin-1 positive; $n=10$ ) (Fig. $6 B, H$ ). Syntenin-1 with mutations in both PDZ domains (G128E/G212D) showed a reduced, although not significant, clustering $(45.2 \pm 12.4 \% ; n=10 ; p=0.1$ ) (Fig. $6 D, H)$. These mutations (G128E and G128E/G212D) by themselves did not affect the synaptic localization of singly expressed 


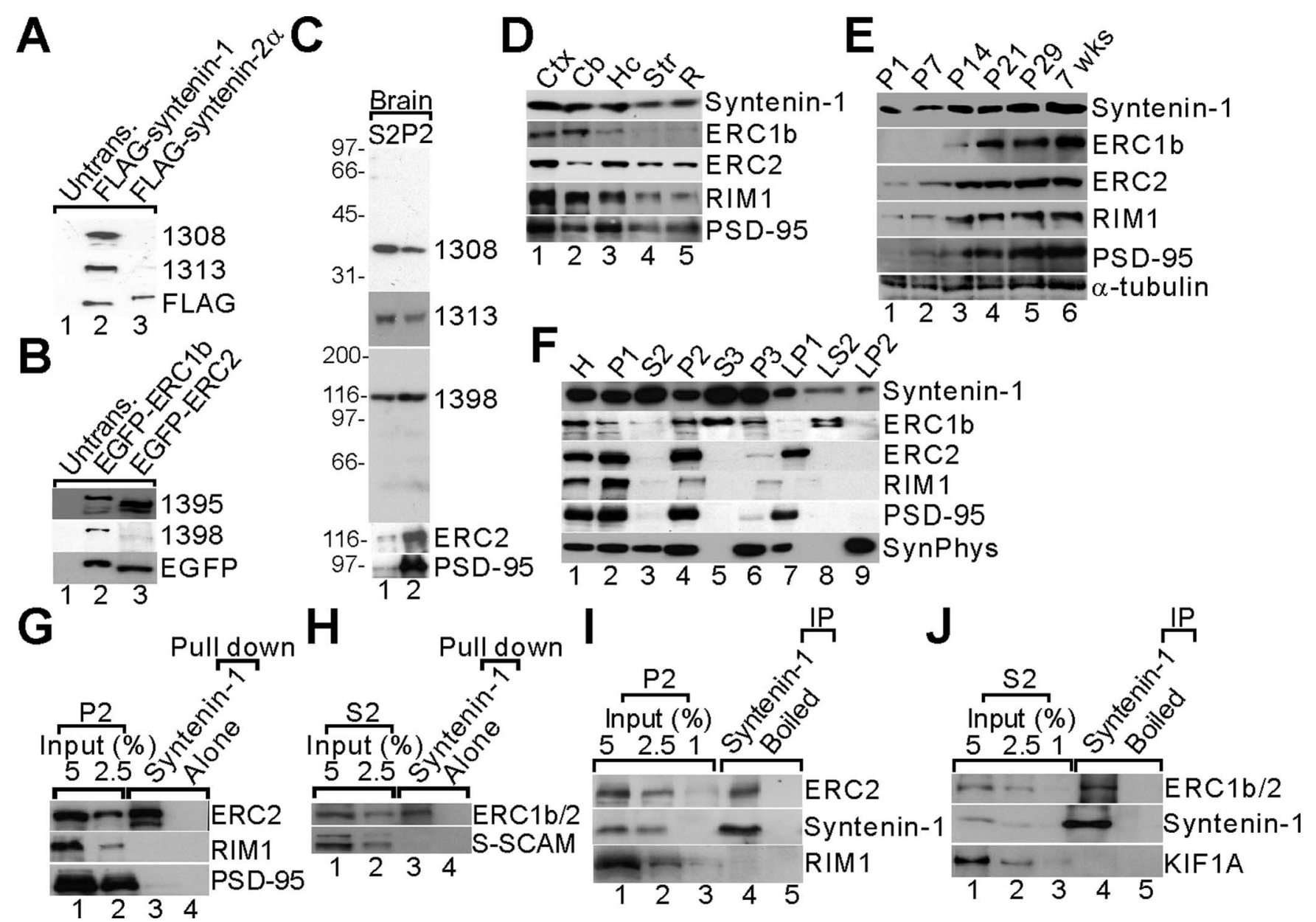

Figure 4. Overlapping expression patterns and biochemical association between ERC and syntenin-1 in the brain. $A-C$, Characterization of ERC and syntenin-1 antibodies. A, Specificity of syntenin-1 antibodies. Lysates of HEK293T cells transfected with FLAG-syntenin-1 or FLAG-syntenin-2 were immunoblotted with syntenin-1 antibodies (1308 and 1313 ). FLAG signals were used for normalization. B, Specificity of ERC1b antibodies. Lysates of HEK293T cells transfected with EGFP-ERC1b or EGFP-ERC2 were immunoblotted with antibodies raised against ERC1b (1395 and 1398). Note that the 1398 antibody is specific for ERC1b, whereas the 1395 antibody recognizes both ERC $1 b$ and ERC2. EGFP signals were used for normalization. C, Recognition of a single target band in rat brain by syntenin-1 and ERC $1 \mathrm{~b}$ antibodies. Rat brain fractions (S2, P2; see below for details) were immunoblotted with the indicated syntenin-1 (1308 and 1313 ) and ERC1b (1398) antibodies. PSD-95 and ERC2 (1292) antibodies were used as controls. $D$, Expression of syntenin-1 and ERC proteins in brain regions. Adult rat brain homogenates were immunoblotted with syntenin-1 and ERC (1398 for ERC1b and 1292 for ERC2) antibodies. RIM1 and PSD-95 antibodies were used as controls. Ctx, Cerebral cortex; Hc, hippocampus; Cb, cerebellum; Str, striatum; R, the rest of the brain. $\boldsymbol{E}$, Postnatal expression of syntenin-1 and ERC in rat brain. $F$, Subcellular distribution of syntenin-1 and ERC. H, Homogenates; P1, nuclei and other large debris; P2, crude synaptosomes; $S 2$, supernatant after $P 2$ precipitation; $S 3$, cytosol; $P 3$, light membranes; LP1, synaptosomal membranes; $L S 2$, synaptosomal cytosol; LP2, synaptic vesicle-enriched fraction; SynPhys, synaptophysin. $\mathbf{G}, \boldsymbol{H}$, Pull down of endogenous ERC2 by syntenin-1. P2 ( $\boldsymbol{G}$ ) and S2 ( $\boldsymbol{H}$ ) adult rat brain lysates were pulled down by GST-syntenin-1 and immunoblotted. ERC1b/2 (1395) antibodies were used for S2 precipitation as ERC 1 b is more abundant in this fraction. $I, J$, In vivo coimmunoprecipitation between ERC and syntenin-1.P2 (I) and S2 $(J)$ adult rat brain lysates were precipitated with syntenin-1 (1308) antibodies and immunoblotted. RIM1 and KIF1A (a motor protein) antibodies were used as controls. Untrans., Untransfected; IP, immunoprecipitated.

syntenin-1 (G128E: $12.4 \pm 4.2 \%$ of Piccolo puncta were syntenin-1 positive, $n=10$; G128E/G212D: $17.0 \pm 4.3 \%, n=12$ ) (Fig. $6 A, C, G)$. These results suggest that, although PDZ2 partly mediates syntenin-1 clustering, mechanisms other than the PDZ interaction are involved in ERC2-dependent syntenin-1 clustering.

How is the syntenin-1 G128E/G212D that lacks ERC2 binding localized to ERC2 clusters? Because syntenin-1, like ERC, forms multimers (Koroll et al., 2001), syntenin-1 G128E/G212D could be recruited to ERC2 clusters by forming multimers with ERC2associated endogenous syntenin-1. To examine this hypothesis, we identified a point mutation (F197R) that disrupts syntenin-1 multimerization (Fig. 6I,J). When F197R was combined G128E/ G212D, the triple syntenin-1 mutant (F197R/G128E/G212D) was primarily diffusely distributed throughout the neuron (Fig. $6 E$ ). Importantly, coexpressed ERC2 could not induce the clustering of this syntenin-1 mutant (Fig. 6F). These results suggest that both PDZ interaction and syntenin-1 multimerization are involved in ERC2-dependent syntenin-1 clustering.

\section{ERC2 clusters distribute to both synaptic and} extrasynaptic sites

A recent report has shown that $\sim 38 \%$ of presynaptic Bassoon clusters are not in contact with dendrites and that $\sim 67 \%$ of the offdendrite Bassoon clusters are FM dye positive, suggesting that they are orphan release sites (Krueger et al., 2003). To determine whether some of the ERC2 clusters in our cultured neurons are not apposed to postsynaptic sites, neurons were costained for ERC2 and PSD-95, an excitatory postsynaptic marker. ERC2 clusters often colocalized with or were closely apposed to PSD-95 clusters, especially when the axons were in apparent contact with dendrites (Fig. 7A). Notably, the axons in PSD-95-lacking regions also showed clear ERC2 clusters along the axons (Fig. 7B), suggesting that they may represent extrasynaptic ERC2 clusters, although some of them may still colocalize with inhibitory synapses. Considering our previous results that $\sim 97 \%$ of exogenous ERC2 clusters are positive for Piccolo (Ko et al., 2003b), these results suggest that some of the extrasynaptic ERC2 clusters represent orphan release sites. 


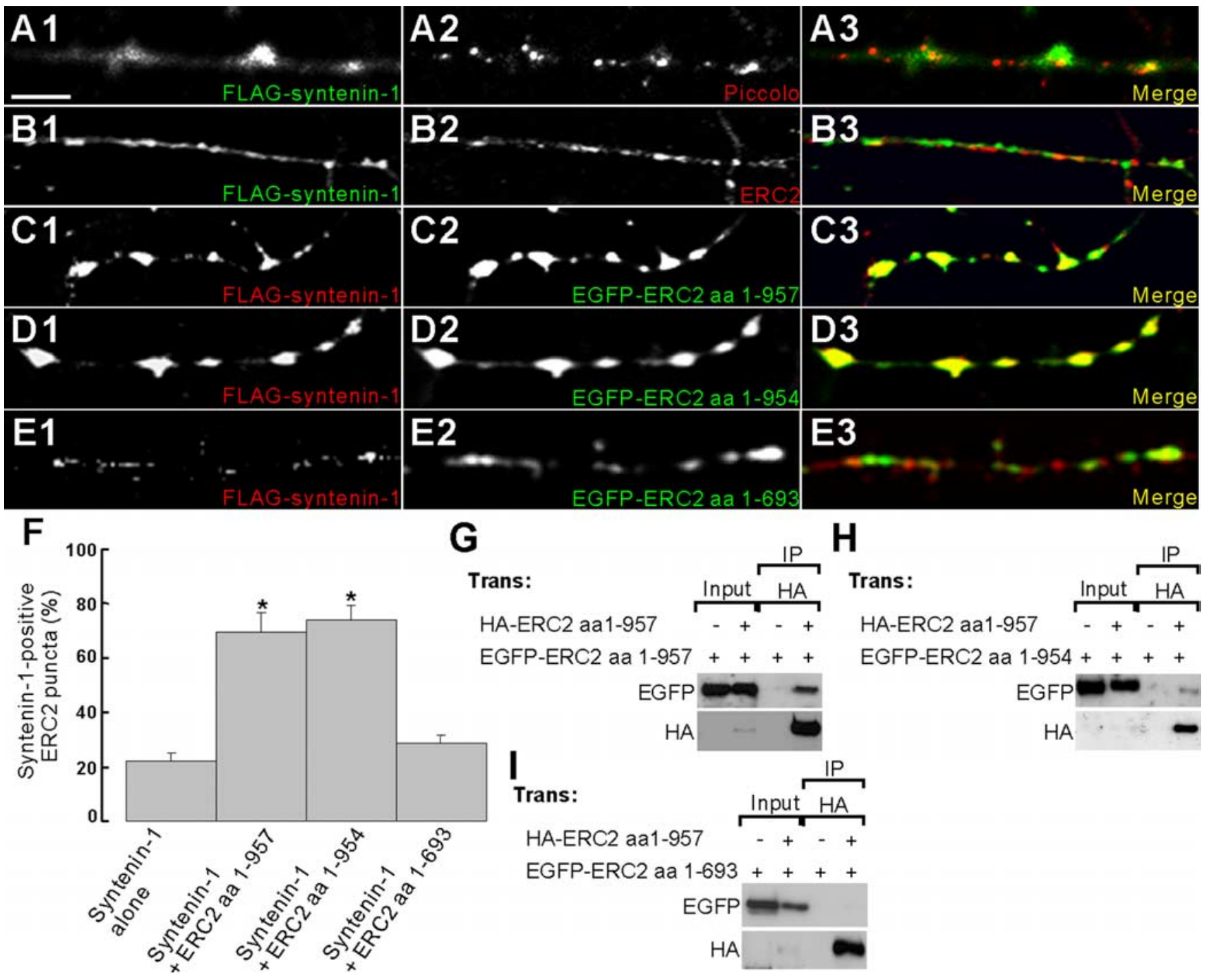

Figure 5. ERC2 promotes presynaptic clustering of syntenin-1 through the PDZ interaction and ERC2 multimerization. $A, B$, Syntenin-1 alone shows a limited synaptic localization. Cultured hippocampal neurons transfected with FLAG-syntenin-1 [7 din vitro (DIV)] were visualized at 9 DIV by immunofluorescence staining for FLAG (syntenin-1) and presynaptic markers (Piccolo or ERC2). Scale bar, $25 \mu \mathrm{m}$. C-E, Full-length ERC2 (amino acids 1-957) and a syntenin-1-binding defective ERC2 variant (amino acids 1-954), but not the ERC2 variant that lacks both syntenin-1 binding and self-multimerization (amino acids 1-693; see below), promote presynaptic syntenin-1 clustering. Neurons transfected with FLAG-syntenin-1 plus EGFP-ERC2 variants (amino acids 1-957, 1-954, or 1-693) were visualized for FLAG (syntenin-1) and EGFP (ERC2). F, Quantitation of ERC2-dependent syntenin-1 clustering. Data are presented as mean \pm SEM. ${ }^{*} p<0.001$. G-I, The C-terminal region of ERC2 (amino acids 694-957) is required for self-multimerization. Lysates of HEK293T cells doubly transfected with HA-ERC2 plus EGFP-ERC2 (amino acids 1-957, 1-954, or 1-693) were precipitated with HA antibodies and immunoblotted with EGFP and HA antibodies. IP, Immunoprecipitated.

\section{Discussion}

PDZ interaction and protein multimerization in ERC2-dependent presynaptic syntenin-1 clustering

Our data indicate that ERC2 promotes presynaptic syntenin-1 clustering through two distinct mechanisms: PDZ interaction and protein multimerization. Syntenin-1 and RIM1, which also binds to ERC2, seem to differ in the degree to which their synaptic targeting depends on the PDZ interaction with ERC2. PDZ deletion in RIM1 incompletely disrupts its synaptic localization (Ohtsuka et al., 2002), suggesting the involvement of additional mechanisms. In contrast, the syntenin-1 mutant lacking both the PDZ interaction and self-multimerization completely loses its presynaptic clustering, suggesting that ERC2 is a key determinant of presynaptic syntenin-1 targeting.

Our results reveal the importance of protein multimerization in ERC2-dependent syntenin-1 clustering. A multimeric ERCsyntenin interaction may have a higher affinity compared with a monomeric interaction and increase both the number and diversity of available protein-protein interaction modules in the ERC2-syntenin-1 complex. Thus, as shown in the heterodimerization-dependent presynaptic targeting of synapsin I (Gitler et al., 2004), protein multimerization may be widely involved in synaptic targeting and assembly.

Roles of the interaction between ERC and syntenin

A relevant in vivo situation for ERC2-dependent syntenin-1 clustering would be the global increase seen in ERC2 expression during postnatal brain development (Fig. $4 E$ ) or the formation of new synaptic contacts between axons and dendrites, in which ERC2 may be accumulated along with other CAZ components (Ziv and Garner, 2004). With regard to the partial synaptic localization of singly expressed syntenin-1, we hypothesize that syntenin-1 is distributed to various subcellular compartments for nonsynaptic functions when synaptic content of ERC2 is low, 

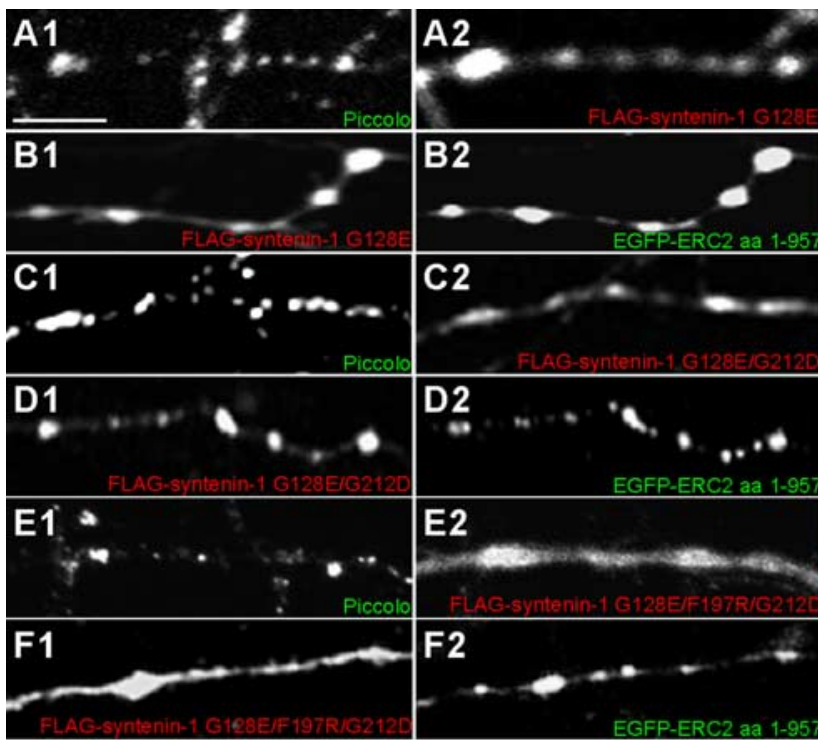

I

Trans:

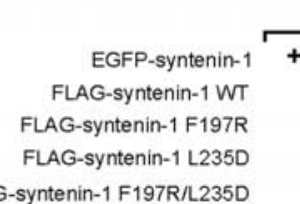

Input (5\%)
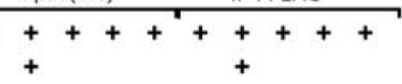

$+$

$+$

$+$

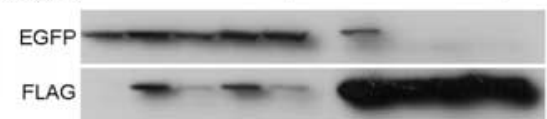

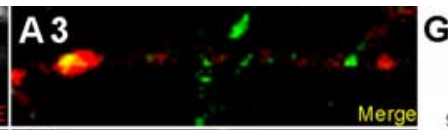

G ${ }^{100}$
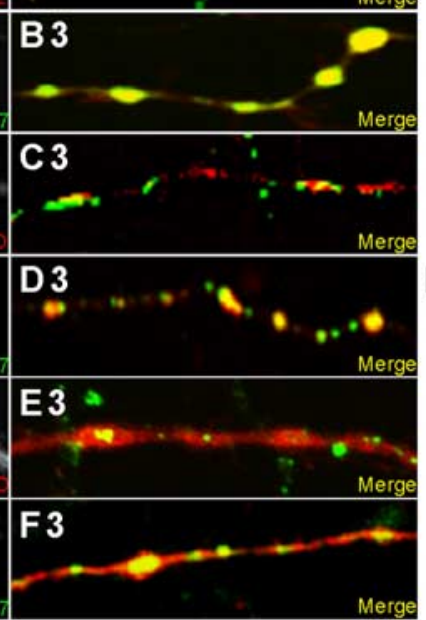

$\mathbf{J}$

Trans:
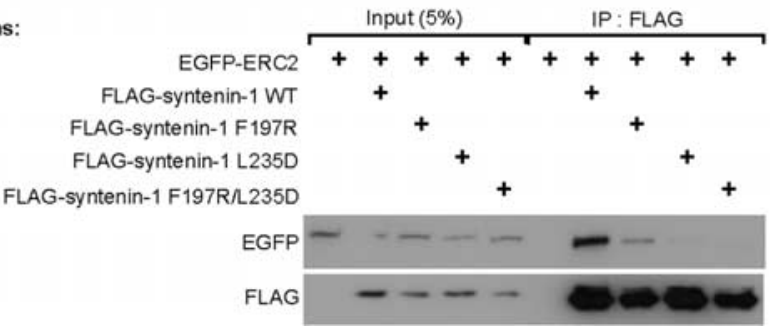

Figure 6. ERC2-dependent syntenin-1 clustering involves syntenin-1 multimerization. $\boldsymbol{A}, \boldsymbol{B}$, Neurons expressing FLAG-syntenin-1 G128E $(\boldsymbol{A})$ or EGFP-ERC2 and FLAG-syntenin-1 G128E ( $\boldsymbol{B})$ were immunostained for Piccolo and FLAG (for single transfection) or FLAG and EGFP (for double transfection). Scale bar, $25 \mu \mathrm{m}$. $\boldsymbol{C}-\boldsymbol{F}$, Similar experiments were performed as in $\boldsymbol{A}$ and $\boldsymbol{B}$ with a FLAG-syntenin-1 G128E/G212D (C, D) and G128E/F197R/G212D (E, F). G, Quantitation of the presynaptic clustering of singly expressed syntenin-1 variants. $\boldsymbol{H}$, Quantitation of the ERC2-dependent clustering of syntenin-1 variants. Data are presented as mean \pm SEM. I Effects of syntenin-1 point mutations on self-multimerization. Lysates of HEK293T cells expressing EGFP-syntenin-1 and FLAG-syntenin-1 point mutants were precipitated with FLAG agarose and immunoblotted with EGFP and FLAG antibodies. Note that both point mutations (F197R, L235D, or F197R/L235D) disrupt syntenin-1 multimerization. Hydrophilic residues ( $R$ and $D$ ) were used for mutation to maximize the solubility of monomerized mutant syntenin-1.J, Effects of syntenin-1 point mutations on ERC2 binding. Note that only syntenin-1 F197R retained the ability to bind ERC2, although to a reduced degree, whereas the other mutants (L235D and F197R/L235D) completely lost the interaction with ERC2, suggesting that the L235D mutation causes nonspecific changes. Wild-type and F197R syntenin-1 bound ERC2 to similar extents in the yeast two-hybrid assay (data not shown), suggesting that the F197R mutation by itself does not reduce the ERC2-syntenin-1 interaction but rather reduces it through mechanisms involving syntenin-1 multimerization. WT, Wild type; Trans, transfected; IP, immunoprecipitated.

whereas a high synaptic content of ERC2 may induce translocation of syntenin-1 to synaptic sites.

The ERC2-syntenin-1 interaction may contribute to the structural organization of active zones. The multimer-based ERC2-syntenin-1 complex may function as a core to which diverse ERC- and syntenin-interacting proteins are recruited. In particular, syntenin-1 may recruit a variety of membrane and cytosolic PDZ ligands including $\beta$-neurexin, a key inducer of presynaptic differentiation (Dean et al., 2003). Of note, syntenin-1 is the only molecule known so far to link $\beta$-neurexin to a CAZ protein, in this case ERC2, suggesting that these interactions may contribute to presynaptic differentiation.

The ERC2-syntenin-1 complex may regulate the function of active zones. The ERC2-RIM1 interaction is known to regulate neurotransmitter release (Takao-Rikitsu et al., 2004). Notably, the dominant-negative reagents used in this study to disrupt the ERC2-RIM1 PDZ interaction are likely to equally disrupt the ERC2-syntenin-1 interaction, which thus may also regulate neurotransmitter release.

ERC2 is mostly active zone specific (Ohtsuka et al., 2002; Wang et al., 2002). In contrast, ERC1b is additionally present in cytosolic and light membrane compartments (Fig. 4F) (Wang et al., 2002), suggesting that the ERC1b-syntenin-1 interaction may occur in these compartments. In this context, it should be noted that ERC1 binds Rab6 (Monier et al., 2002), a small GTPase implicated in post-Golgi traffic in neurons. In addition, syntenin interacts with Rab5 (Tomoda et al., 2004), a small GTPase that regulates synaptic vesicle cycling (Sudhof, 2004). Thus, the ERC1b-syntenin interaction may regulate the trafficking of ERC- and syntenin-associated protein complexes or small membranes. The increase in the number of membrane structures in dendrites of syntenin-overexpressing neurons (Hirbec et al., 2005) may reflect altered protein and membrane trafficking.

Our data indicate that some of the ERC2 clusters in cultured neurons are not apposed to postsynaptic sites. Because most ERC2 clusters are Piccolo positive (Ko et al., 2003b) and a significant fraction of Bassoon clusters that are not in contact with dendrites are FM dye positive ( $\sim 67 \%)$ (Krueger et al., 2003), it is likely that the extrasynaptic ERC2 clusters represent orphan release sites. In this context, it is possible that the ERC2-syntenin-1 interaction contributes to the organization of not only active zones but also orphan release sites. 

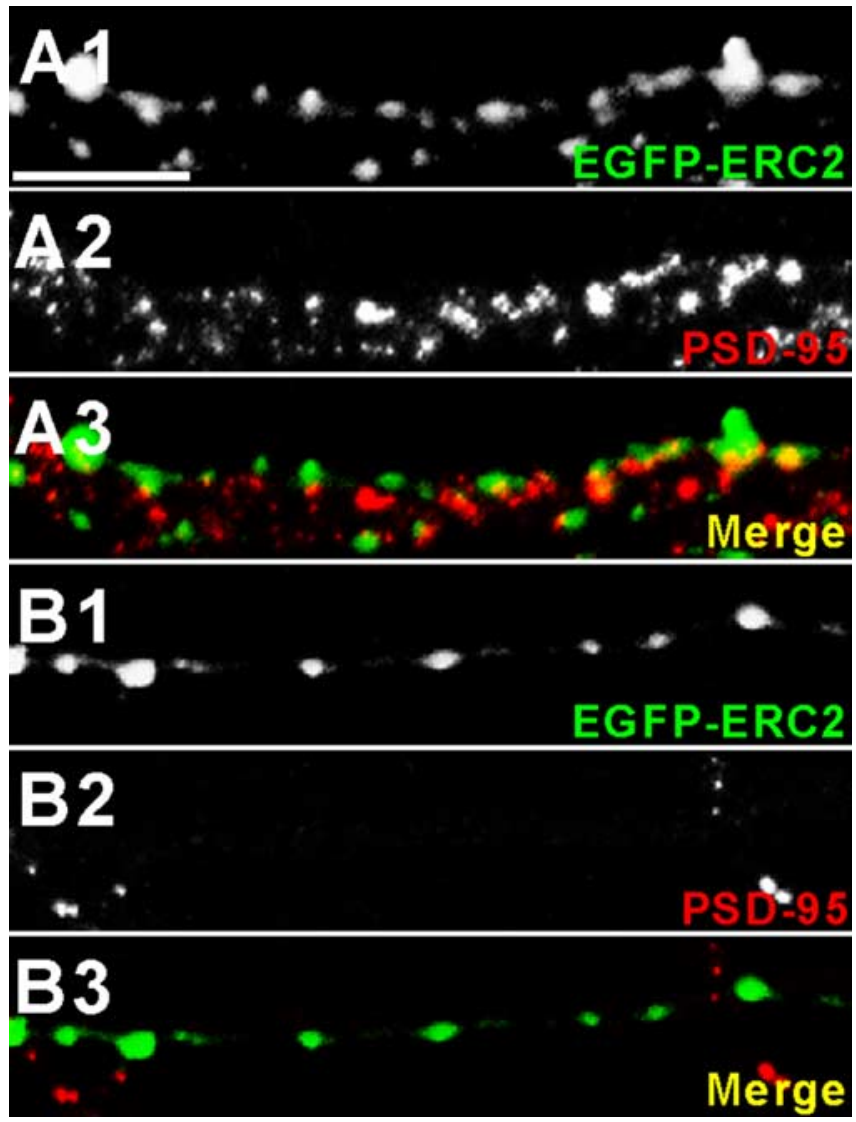

Figure 7. Partial colocalization of ERC2 clusters with PSD-95. A, B, Colocalization or close apposition of ERC2 clusters and PSD-95. Cultured hippocampal neurons transfected with EGFPERC2 [13 $\mathrm{d}$ in vitro (DIV)] were double stained for EGFP (ERC2) and PSD-95 at 15 DIV. Representative ERC2 clusters from PSD-95 cluster-rich $(\boldsymbol{A})$ and PSD-95-lacking $(\boldsymbol{B})$ regions. Scale bar, $25 \mu \mathrm{m}$.

\section{References}

Biederer T, Sara Y, Mozhayeva M, Atasoy D, Liu X, Kavalali ET, Sudhof TC (2002) SynCAM, a synaptic adhesion molecule that drives synapse assembly. Science 297:1525-1531.

Dean C, Scholl FG, Choih J, DeMaria S, Berger J, Isacoff E, Scheiffele P (2003) Neurexin mediates the assembly of presynaptic terminals. Nat Neurosci 6:708-716.

Deguchi-Tawarada M, Inoue E, Takao-Rikitsu E, Inoue M, Ohtsuka T, Takai Y (2004) CAST2: identification and characterization of a protein structurally related to the presynaptic cytomatrix protein CAST. Genes Cells 9:15-23.

Dresbach T, Qualmann B, Kessels MM, Garner CC, Gundelfinger ED (2001) The presynaptic cytomatrix of brain synapses. Cell Mol Life Sci 58:94-116.

Enz R, Croci C (2003) Different binding motifs in metabotropic glutamate receptor type $7 \mathrm{~b}$ for filamin $\mathrm{A}$, protein phosphatase $1 \mathrm{C}$, protein interacting with protein kinase C (PICK) 1 and syntenin allow the formation of multimeric protein complexes. Biochem J 372:183-191.

Gitler D, Xu Y, Kao HT, Lin D, Lim S, Feng J, Greengard P, Augustine GJ (2004) Molecular determinants of synapsin targeting to presynaptic terminals. J Neurosci 24:3711-3720.

Grootjans JJ, Zimmermann P, Reekmans G, Smets A, Degeest G, Durr J, David G (1997) Syntenin, a PDZ protein that binds syndecan cytoplasmic domains. Proc Natl Acad Sci USA 94:13683-13688.

Hirbec H, Perestenko O, Nishimune A, Meyer G, Nakanishi S, Henley JM, Dev KK (2002) The PDZ proteins PICK1, GRIP, and syntenin bind multiple glutamate receptor subtypes. Analysis of PDZ binding motifs. J Biol Chem 277:15221-15224.

Hirbec H, Francis JC, Lauri SE, Braithwaite SP, Coussen F, Mulle C, Dev KK, Coutinho V, Meyer G, Isaac JT, Collingridge GL, Henley JM, Couthino V
(2003) Rapid and differential regulation of AMPA and kainate receptors at hippocampal mossy fibre synapses by PICK1 and GRIP. Neuron 37:625-638.

Hirbec H, Martin S, Henley JM (2005) Syntenin is involved in the developmental regulation of neuronal membrane architecture. Mol Cell Neurosci 28:737-746.

Kim D, Kim EH, Kim C, Sun W, Kim HJ, Uhm CS, Park SH, Kim H (2003) Differential regulation of metallothionein-I, -II, and -III mRNA expression in the rat brain following kainic acid treatment. NeuroReport 14:679-682.

Kim E, Sheng M (2004) PDZ domain proteins of synapses. Nat Rev Neurosci 5:771-781.

Kim S, Ko J, Shin H, Lee JR, Lim C, Han JH, Altrock WD, Garner CC, Gundelfinger ED, Premont RT, Kaang BK, Kim E (2003) The GIT family of proteins forms multimers and associates with the presynaptic cytomatrix protein Piccolo. J Biol Chem 278:6291-6300.

Ko J, Kim S, Valtschanoff JG, Shin H, Lee JR, Sheng M, Premont RT, Weinberg RJ, Kim E (2003a) Interaction between liprin- $\alpha$ and GIT1 is required for AMPA receptor targeting. J Neurosci 23:1667-1677.

Ko J, Na M, Kim S, Lee JR, Kim E (2003b) Interaction of the ERC family of RIM-binding proteins with the liprin-alpha family of multidomain proteins. J Biol Chem 278:42377-42385.

Koroll M, Rathjen FG, Volkmer H (2001) The neural cell recognition molecule neurofascin interacts with syntenin- 1 but not with syntenin-2, both of which reveal self-associating activity. J Biol Chem 276:10646-10654.

Krueger SR, Kolar A, Fitzsimonds RM (2003) The presynaptic release apparatus is functional in the absence of dendritic contact and highly mobile within isolated axons. Neuron 40:945-957.

Mok H, Shin H, Kim S, Lee JR, Yoon J, Kim E (2002) Association of the kinesin superfamily motor protein $\mathrm{KIF} 1 \mathrm{~B} \alpha$ with postsynaptic density-95 (PSD-95), synapse-associated protein-97, and synaptic scaffolding molecule PSD-95/discs large/zona occludens-1 proteins. J Neurosci 22:5253-5258.

Monier S, Jollivet F, Janoueix-Lerosey I, Johannes L, Goud B (2002) Characterization of novel Rab6-interacting proteins involved in endosome-toTGN transport. Traffic 3:289-297.

Nakata T, Kitamura Y, Shimizu K, Tanaka S, Fujimori M, Yokoyama S, Ito K, Emi M (1999) Fusion of a novel gene, ELKS, to RET due to translocation $\mathrm{t}(10 ; 12)(\mathrm{q} 11 ; \mathrm{p} 13)$ in a papillary thyroid carcinoma. Genes Chromosomes Cancer 25:97-103.

Ohno K, Koroll M, El Far O, Scholze P, Gomeza J, Betz H (2004) The neuronal glycine transporter 2 interacts with the PDZ domain protein syntenin-1. Mol Cell Neurosci 26:518-529.

Ohtsuka T, Takao-Rikitsu E, Inoue E, Inoue M, Takeuchi M, Matsubara K, Deguchi-Tawarada M, Satoh K, Morimoto K, Nakanishi H, Takai Y (2002) Cast: a novel protein of the cytomatrix at the active zone of synapses that forms a ternary complex with RIM1 and munc13-1. J Cell Biol 158:577-590.

Rosenmund C, Rettig J, Brose N (2003) Molecular mechanisms of active zone function. Curr Opin Neurobiol 13:509-519.

Shin H, Wyszynski M, Huh KH, Valtschanoff JG, Lee JR, Ko J, Streuli M, Weinberg RJ, Sheng M, Kim E (2003) Association of the kinesin motor KIF1A with the multimodular protein liprin-alpha. J Biol Chem 278:11393-11401.

Sudhof TC (2004) The synaptic vesicle cycle. Annu Rev Neurosci 27:509-547.

Takao-Rikitsu E, Mochida S, Inoue E, Deguchi-Tawarada M, Inoue M, Ohtsuka T, Takai Y (2004) Physical and functional interaction of the active zone proteins, CAST, RIM1, and Bassoon, in neurotransmitter release. J Cell Biol 164:301-311.

Tomoda T, Kim JH, Zhan C, Hatten ME (2004) Role of Unc51.1 and its binding partners in CNS axon outgrowth. Genes Dev 18:541-558.

Wang Y, Liu X, Biederer T, Sudhof TC (2002) A family of RIM-binding proteins regulated by alternative splicing: implications for the genesis of synaptic active zones. Proc Natl Acad Sci USA 99:14464-14469.

Zhen M, Jin Y (2004) Presynaptic terminal differentiation: transport and assembly. Curr Opin Neurobiol 14:280-287.

Ziv NE, Garner CC (2004) Cellular and molecular mechanisms of presynaptic assembly. Nat Rev Neurosci 5:385-399. 